\title{
The epithelial-mesenchymal interactions: insights into physiological and pathological aspects of oral tissues
}

\author{
Arvind Babu Rajendra Santosh, Thaon Jon Jones \\ The University of the West Indies, Mona, Kingston, Jamaica
}

\begin{abstract}
In the human biological system, the individual cells divide and form tissues and organs. These tissues are hetero-cellular. Basically any tissue consists of an epithelium and the connective tissue. The latter contains mainly mesenchymally-derived tissues with a diversified cell population. The cell continues to grow and differentiate in a pre-programmed manner using a messenger system. The epithelium and the mesenchymal portion of each tissue have two different origins and perform specific functions, but there is a well-defined interaction mechanism, which mediates between them. Epithelial mesenchymal interactions (EMIs) are part of this mechanism, which can be regarded as a biological conversation between epithelial and mesenchymal cell populations involved in the cellular differentiation of one or both cell populations. EMIs represent a process that is essential for cell growth, cell differentiation and cell multiplication. EMIs are associated with normal physiological processes in the oral cavity, such as odontogenesis, dentino-enamel junction formation, salivary gland development, palatogenesis, and also pathological processes, such as oral cancer. This paper focuses the role EMIs in odontogenesis, salivary gland development, palatogenesis and oral cancer.
\end{abstract}

\section{Introduction}

Epithelial mesenchymal interactions (EMIs) are described as a series of programmed, sequential and reciprocal (complex and multiphase) communications between the epithelium and the mesenchyme with its heterotypic cell population, that result in the differentiation of

Correspondence: Arvind Babu Rajendra Santosh, The University of the West Indies, Mona, Kingston, Jamaica.

E-mail: arvindbabu2001@gmail.com

Key words: epithelial mesenchymal interactions, odontogenesis, dentinoenamel junction, salivary gland development, palatogenesis, tumorogenesis.

Received for publication: 22 November 2013.

Revision received: 20 January 2014.

Accepted for publication: 26 February 2014.

This work is licensed under a Creative Commons Attribution NonCommercial 3.0 License (CC BY-NC 3.0).

(C) Copyright A.B. Rajendra Santosh and T.J. Jones., 2014

Licensee PAGEPress, Italy

Oncology Reviews 2014; 8:239

doi:10.4081/oncol.2014.239 one or both cell populations. ${ }^{1}$ This growth and development process requires an understanding and an interaction between homotypic and/or heterotypic cell populations on the molecular level. Odontogenesis is the process of tooth development, which involves both ectodermal and mesenchymal components, being the key elements in the development of teeth. In order for the tooth to form, an interactive mechanism between these heterotypic cellular populations is required. ${ }^{2}$ EMI mechanisms can be observed in the dentino-enamel junction of the tooth. Glands result from the modification of the epithelial structure. During the development of salivary glands, there is a similar interaction between the epithelium and the mesenchyme of this anatomical site. ${ }^{3}$ EMI mechanisms can be observed in the development of palatal shelves in palatogenesis. Tumorigenesis is considered to be a complex process of abnormal cell formation. Cancer originates from a single cell, which seems to be of monoclonal nature and is surrounded by other normal and non-mutated cells. Once a malignant tumor is established, the inherent genetic instability of multiple tumor cells could favor the development of sub-populations and clones, that can lead to the expansion of a polyclonal tumor cell population. ${ }^{4,5}$ Ingber et al. in 2002 argued that, from the histogenetic perspective, the epithelial-mesenchymal interactions and the resulting deposition of the extracellular matrix may actively contribute to the carcinogenic process. ${ }^{6}$ Del Moral et al. in 2010 stated that the roles of the epithelium and the mesenchyme in this signaling mechanism can be determined using studies on recombinant. ${ }^{7}$ Hence the interaction and signaling mechanism between the epithelial tissue and the mesenchymal tissue (two heterotypic tissues) plays a major role in the formation of a tumor. A greater understanding of the EMIs in these physiological processes may enable us to gain new insights on the mechanism and the molecules involved, and more knowledge about the EMI process in tumorigenesis. Further understanding of EMIs in both physiological and pathological processes will provide us with a deeper understanding of the flexibility of cellular phenotypes and eventually open up new research avenues in the development of new treatment strategies.

\section{Epithelial mesenchymal interactions in odontogenesis}

Odontogenesis can be described as a complex physiological process of tooth development from ectodermal and mesodermal appendages. Interactions between the ectodermal tissue and the underlying mesenchymal tissue are at the basis of the central mechanism regulating the morphogenesis of teeth. Tooth morphogenesis is a progressive process regulated by sequential and reciprocal interactions between epithelial and mesenchymal tissues, during which the simple oral ectoderm thickens, buds, grows and folds to form the complex shape of the tooth crown. The morphological classification system of tooth 
development divides these changes into stages, namely bud, cap and bell stages. The structures involved in the stages of tooth development include the enamel organ, the dental papillae and the dental sac. The enamel organ derives from the ectoderm, whereas the dental papillae and the dental sac are of mesodermal origin. The enamel organ is composed of ameloblasts, the stellate reticulum and the cells of the stratum intermedium. The dental papillae consist of odontoblasts and mesenchymal cells. For the development of teeth, the interaction between these ectodermal and mesodermal tissues is essential. In 1887, Von Brunn suggested that odontoblasts differentiate only in the presence of the enamel epithelium. ${ }^{8}$ The specific features of the EMIs are explained by the studies on recombinant DNA, which focus on the combination of two or more sources of tissues/DNA. These studies often involve the combination of DNA from different organisms and depend on the ability to cut and recombine DNA molecules at certain points identified by specific sequences of nucleotide bases. ${ }^{9}$ In 2010 , Del Moral et al. maintained that the roles of the epithelium and the mesenchyme in these signaling process can be determined using studies on recombinant tissues. ${ }^{7}$ These studies have shown that epithelial signals induced in the mesenchyme lead to the subsequent development of the tooth. The proliferative response of epithelial cells via the keratinocyte growth factor was investigated by studies on recombinant tissues, which provided some evidence that was used to study the EMIs. ${ }^{10,11}$ The experimental procedures in these studies are based on a culture of the bud stage of the tooth development process to evaluate the role of EMI molecules. ${ }^{2}$ In 2003, Garant reported that the results from recombinant studies revealed that the tooth continuous to develop in the following two combinations: i) dental epithelium with dental mesenchyme and ii) dental epithelium and skin mesenchyme. On the contrary, no tooth development occurred in cultures obtained with the dental epithelium only, i) the dental mesenchyme, ii) the skin epithelium with dental mesenchyme. ${ }^{12}$ Lesot claimed that EMIs are mediated by the extracellular matrix components of the basement membrane, which contains the signaling molecules and enables a strict time and space regulation of cell kinetics, epithelial histogenesis and morphogenesis. ${ }^{13}$

In 2008, Li et al. reported that the time and space distribution patterns of the transforming growth factor (TGF)- $\beta 2$, especially in the basement membrane, the epithelial cells in the basal layer, the enamel knot, the mature odontoblast and ameloblast pointed to a close association between TGF- $\beta 2$ signaling and tooth crown development, thus indicating that the TGF- $\beta 2$ has a role in tooth initiation and epithelial morphogenesis. ${ }^{14}$

In 2013, Angelova Volponi et al. in their bioengineering studies used the adult human gingival cells and the rat dental mesenchyme to develop the tooth. They concluded that epithelial cells derived from adult gingival tissue are capable of responding to tooth-inducing signals from the embryonic tooth mesenchyme and contribute to the development of the tooth. ${ }^{15}$ A recent bioengineering study considered the dental epithelium and the dental mesenchyme as a model contributing to tooth development, as reported by Garant in 2003. A novel area in this recent bioengineering study is the use of the human gingival epithelium with the rat mesenchyme. ${ }^{12}$ These results showed that time and space specific information is encoded in the basement membrane components. The messenger and signaling information is read by the cell membrane receptor molecules of the adjacent cells. ${ }^{16,17}$ It is clearly understood that, for EMIs to occur, some form of messenger system is required between the epithelium and the connective tissue. ${ }^{18}$ These studies also showed that the extracellular matrix molecules and some specific genes mediate these EMI mechanisms. For the EMIs to occur, some form of the messenger system is also required between the epithelium and the mesenchyme. The messenger system could be anyone of the following seven: ${ }^{19,20}$ i) direct cell-cell communication involving the cytoplasmic process and gap junctions; ii) matrix vesicles between two cell populations; iii) ions like potassium $\left(\mathrm{K}^{+}\right)$and calcium $\left(\mathrm{Ca}^{+2}\right)$ ions; iv) extracellular matrix (ECM) molecules like collagen IV, I, III and fibronectin, tenascin, epithelial cadherin (E-cadherin), laminin; v) molecular diffusion of hormones and growth factors, such as the bone morphogenetic factor, the fibroblast growth factor, the epidermal growth factor, and the TGF; vi) autocrine and paracrine regulators; and vii) messenger RNA. ${ }^{6,19,20}$ More than 250 genes are reported to be involved in tooth development and control number, position, size and shape of teeth. ${ }^{21}$ The majority of these genes are associated with the central regulations of tooth development and also with interactions of the signaling pathways between cells and tissues. The genes regulate the mediators for the signaling pathways and the interaction mechanism. In 2003, Irma Thesleff reported the genes involved in signaling tooth development: pitx2, p21, Msx2, Lef1, Edar, Lhx6, Lhx7, Dix1, Dix2, Paz9, Gli1, Gli2, Gli3, Barxl and Runx2. These genes have a role in the differentiation and the mineralization during the tooth development process. ${ }^{2}$ Therefore EMIs in odontogenesis are considered to be a programmed series of events, which are controlled by various genes, growth factors and extracellular molecules. Hierarchical and sequential functional interactions between the epithelium and the mesenchyme are considered to be a hallmark of embryonic development. ${ }^{22,23}$

\section{Epithelial mesenchymal interactions in dentino- enamel junction}

The enamel originates from the epithelial tissue, and the enamelforming cell is called ameloblast. On the contrary, dentine derives from the mesenchymal tissue, and the dentine-forming cell is called odontoblast. The location where the enamel and the dentine join is called dentino-enamel junction. The interdigitation of the ameloblasts and odontoblasts is essential for the union of this heterotypic cell population. ${ }^{24}$

\section{Epithelial mesenchymal interactions in salivary gland development}

A gland is a modification of the epithelial structure, and forms to carry out specific functions.$^{25} \mathrm{~A}$ salivary gland is also an epithelial modification and is made up of epithelial components, such as acinar and ductal cells, mesenchymal structures, such as fibrous stroma, vascular elements and a fibrous capsule. Hence EMIs are required for the development of the salivary gland tissue. In 2007, Tucker maintained that the stages in the salivary gland development process are pre-bud, initial bud, pseudo-glandular, canalicular and terminal bud. ${ }^{26}$ These developmental stages of the salivary gland formation suggests that branching occurs first, whereas the acinar cytodifferentiation occurs at later stages. Hence, the role of EMIs in salivary gland formation will be explained in both the branching pattern of the gland's formation and the acinar cytodifferentiation.

The presence of EMIs is shown in the studies on recombinant tissues based on cultured combinations of the salivary gland epithelium from the initial bud stage with either the mandibular arch mesenchyme, the mammary gland mesenchyme, or the lung mesenchyme. These combinations showed no normal salivary gland branching pattern. Conversely, culturing combinations of salivary gland mesenchyme with the epithelium of either mammary gland, oral, nasal, or early pituitary gland epithelium showed normal salivary gland like branching. ${ }^{3}$ 
The experiments reveal that the mesenchymal tissue from the anatomical location of the salivary gland has a greater role than the branching pattern of the gland. Further culturing combinations of salivary gland epithelium from the later stages to the initial bud stage with lung mesenchyme showed a normal branching pattern of the gland. ${ }^{3}$ These experiments suggest that messenger molecules are already incorporated into the salivary gland epithelium due to the timing in the development process and could follow the normal salivary gland branching pattern, even though it is cultured with lung mesenchyme. ${ }^{3}$

In other experimental studies, cultured combinations of submandibular salivary gland mesenchyme and parotid epithelium revealed amylase-secreting serous acinar cells development. Experiments on cultures with a combination of submandibular salivary gland mesenchyme and parotid epithelium revealed the branching of the sub-mandibular salivary gland and an epithelium that could synthesize milk protein after stimulation. ${ }^{27}$ In contrast, recombinant studies that cultured combinations of early pituitary epithelium and submandibular salivary gland mesenchyme reported the expression of amylase, whereas culturing combinations of later stages of pituitary epithelium with submandibular salivary gland mesenchyme showed a loss in the flexibility of the amylase expression. The result of this study suggests that EMIs have a time-based role in the development of the salivary gland. ${ }^{28}$

In 1980, Cutler stated on the basis of his experimental study on the salivary gland tissue that, in the development of the sub-mandibular salivary gland, morphogenesis and cytodifferentiation are partially coupled, yet independently regulated processes. The earliest phases of morphogenesis (rudiment down growth and primary branching) seem to be required to initiate cytodifferentiation. Once initiated, cytodifferentiation proceeds with continued morphogenesis (tissue organization) or significant amounts of connective tissue elements. ${ }^{29}$

Recombinant studies with salivary glands and the role of EMIs suggest that the branching pattern can be altered by the mesenchyme, but the cytodifferention of the salivary gland epithelial cells cannot be altered, and conclude that cytodifferentiation is an innate characteristic of the epithelium. Terminal differentiation (acinar cytodifferentiation) of the salivary gland epithelium is not dependent on branching, therefore, even when branching is blocked, the cell continues to undergo differentiation. In summary, differentiation is not dependent on signals from the mesenchyme, but it is the isolated epithelium that forms the secretory granules. It should also be noted that temporal and spatial relations were also observed in the development of salivary gland tissue.

\section{Epithelial mesenchymal interactions in palatogenesis}

Palatogenesis is the embryogenic developmental process of the palate. When the palate is well-developed, it separates the nasal and the oral cavities. During the $6^{\text {th }}$ week of palatogenesis, the palatal shelves grow rapidly along the vertical plane by proliferating mesenchymal cells, which undergo a sudden elevation to bring them into a horizontal apposition above the flattening tongue. The movement from the vertical to the horizontal plane of the palate results from the flattening of the tongue during glossogenesis by intrinsic forces and glycosaminoglycans. ${ }^{30}$ Following the horizontal elevation of the palatal shelves, the opposing palatal shelves fuse along the midline through a series of interactions in cell adhesion molecules. This is considered to be the EMIs in palatogenesis. The medial edge epithelia needs to be disintegrated and the acquisition of the mesenchymal property is required for palatal shelves adherence. During this process, the medial edge epithe- lia cells downregulate their E-cadherin, desmosomes, keratin and syndecan; a simultaneous upregulation of vimentin has also been observed. ${ }^{31}$ Thus EMIs enable epithelial mesenchymal transitions (EMTs), promoting palatal shelves adherence.

\section{Epithelial mesenchymal interactions in oral cancer}

Tumorigenesis is the process of cancer formation, and the newlyformed mass of tissue is termed neoplasia (neo: new; plasis: growth). It is well-documented in the literature that the neoplastic tissue is formed as a result of abnormal and dysregulated genetic material. The role of EMIs in cancer can be better explained by using the example of the epithelial tissue malignancies. The new mass of tissue requires angiogenesis for their sustained proliferation and growth. ${ }^{5}$ Therefore the role of the stromal microenvironment in angiogenesis is very important in tumor formation. As a consequence of these requirements, epithelial and mesenchymal cells need to have an interactionary signaling mechanism for their growth and proliferation.

\section{Epithelial mesenchymal interactions in normal and malignant epithelium}

The formation of the epithelial tissue is determined by complex interactions between the epithelium itself and its underlying mesenchyme. In 1967, Grobstein stated that one of the key products of EMIs is the accumulation of a specialized ECM scaffold, called basement membrane, along the epithelial-mesenchymal interface. ${ }^{32}$ The basement membrane functions as an extracellular complex of signaling molecules, that drives the differentiation, growth and mitotic signaling of adjacent adherent cells, in addition to stabilizing the character of the epithelium. Therefore the basement membrane can be said to maintain the normal histogenesis and morphogenesis of the epithelium. In the case of malignant tissue, where there is loss of basement membrane contiguity, normal histogenesis is lost; and the involved epithelial tissue adversely proliferates. Most tumors are involved in deregulated interactions between epithelial cells and subjacent mesenchymally-derived connective tissue stroma.

During the oncogenic change, the malignant epithelial tissue gains the ability to induce angiogenesis from the surrounding stroma, thus explaining the importance of the role of EMIs in cancer. As a result, the concept of angiogenesis in tumorigenesis further reinforces the role of EMIs in cancer.

\section{Epithelial mesenchymal interaction and epithelial mesenchymal transition}

Epithelial malignancies can be microscopically evaluated based on the anatomic integrity of the basement membrane. It is clearly understood that any breach or break in the basement membrane continuity is an obvious indication of frank malignancy. This can occur due to a loss of cell adhesion between the mesenchymal structure and the membrane. This loss of cell adhesion is considered to be a hallmark of malignancy. ${ }^{33}$ At the molecular level of cell adhesion, this loss is due to the E-cadherin deregulation, which results in the dissolution of cell-cell tight junctions, and at the same time to the upregulation of a number 
of mesenchymal markers, including neural cadherin (N-cadherin), vimentin and fibronectin. ${ }^{34}$ The cadherin switch from E-cadherin to $\mathrm{N}$ cadherin is considered a hallmark of the epithelial-mesenchymal transition and progression of carcinomas. ${ }^{35}$ At this stage, the transformed cell is said to be acquiring mesenchymal properties and therefore to be a hallmark of the EMTs. Furthermore, there is a morphogenetic acquisition of spindle-shaped cells coupled with a loss of polarity in transformed cell. The new physiologic make-up of the cell setting promotes: cell migration, increased metastatic behavior, drug resistance and cancer stem cell transformation. Therefore EMIs and EMTs are the key biological alterations that define the malignant character of cells. This can be often seen microscopically as the invasive front of the tumor tissue.

EMIs may also contribute to tumor initiation in the oral cavity. For instance, the chemical carcinogenesis of the epithelium requires the presence of closely-apposed carcinogen treated epithelium. In 1966 Dawe et al. suggested that the malignant transformation cannot take place in an isolated submandibular epithelium or mesenchyme, even though the resulting tumor is epithelial in origin. ${ }^{36}$ In 1981, Goldenberg et al. in their mice study suggested that epithelial tumors also recruit normal stromal cells to become tumorigenic. ${ }^{37}$ The progression of cancer from cell transformation relies on the communication between the tumor cells and the stromal microenvironment. ${ }^{38,39}$ In contrast, few studies with a combination of various unorganized epithelial cancers with normal embryonic mesenchyme showed a reversal of the malignant phenotype, as evidenced by the restoration of the normal epithelial organization and histodifferentiation. ${ }^{40}$

\section{Conclusions}

Epithelial mesenchymal interactions appear to play an important role in the formation and the maintenance of normal tissue architecture, whereas their dysregulation contribute to tumorigenesis. The role of EMIs can be best explained in oral tissues, due to the higher epithelial contribution to the development of oral anatomical structures. The role of EMIs in cancer is clearly understood in epithelial malignancies, due to the function of the basement membrane and the required angiogenic process in cancer. Since epithelial malignancies are more common in oral cancer, the roles of EMIs are better investigated in oral specimens. The test laboratories that diagnose oral cancer need now to research the cause of the alterations in the basement membrane and probe the molecular elements related to the basement membrane. Research should also include the molecules in the tumor microenvironment. A statistical correlation must be identified between molecular elements and parameters such as tumor initiation, progression, angiogenesis, invasion and metastasis. This should lead to a greater understanding of the role of EMI and EMT molecules in tumorigenesis and to the future development of molecular therapeutic solutions for oral cancer.

\section{References}

1. Manjunatha BS, Kumar GS. Epithelial mesenchymal interactions in odontogenesis. J Oral Maxillofac Pathol 2005;9:51-7.

2. Thesleff I. Epithelial-mesenchymal signalling regulating tooth morphogenesis. J Cell Sci 2003;116:1647-8.

3. Ball WD. Development of the rat salivary glands. 3. Mesenchymal specificity in the morphogenesis of the embyronic submaxillary and sublingual glands of the rat. J Exp Zool 1974;188:277-88.

4. Alison MR, Lim SM, Nicholson LJ. Cancer stem cells: problems for therapy? J Pathol 2011;223:147-61.
5. Cotran RS, Kumar V, Collins T, Robbins SL. Pathologic basis of disease. Neoplasia. 8th ed. Philadelphia: Elseiver Publ.; 2010. pp 259330.

6. Ingber DE. Cancer as a disease of epithelial-mesenchymal interactions and extracellular matrix regulation. Differentiation 2002;70: $547-60$.

7. Del Moral PM, Warburton D. Explant culture of mouse embryonic whole lung, isolated epithelium, or mesenchyme under chemically defined conditions as a system to evaluate the molecular mechanism of branching morphogenesis and cellular differentiation. Methods Mol Biol 2010;633:71-9.

8. Diekwisch TG. The developmental biology of cementum. Int J Dev Biol 2001;45:695-706.

9. Pandey S, Suba N. Recombinant DNA technology and genetic engineering: a safe and effective meaning for production valuable biologicals. Int J Pharmaceutical Sci Rev Res 2010;1:14-20.

10. Goldberg ID, Rosen EM, eds. Epithelial mesenchymal interactions in cancer. Basel, Boston, Belin: Birkhauser Publ.; 1995. pp 202-5.

11. Glasstone $\mathrm{S}$. The development of tooth germs in vitro. J Anat 1983;70:260-66.

12. Garant PR. Epithelial mesenchymal morphogenic regulation of odontogenesis. In: Garant PR, ed. Oral cells and tissues. Chicago, IL: Quintessence Publ.; 2003. pp 7-11.

13. Lesot H. Tooth engineering. Regenerative manomedicine. Available from: http://www.regmed.fr/4213-2/

14. Li JY, Hu B, Wang XJ, Wang SL. Temporal and spatial expression of TGF-beta2 in tooth crown development in mouse first lower molar. Eur J Histochem 2008;52:243-50.

15. Angelova Volponi A, Kawasaki M, Sharpe PT. Adult human gingival epithelial cells as a source for whole-tooth bioengineering. J Dent Res 2013;92:329-34.

16. Ruch JV, Lesot H, Karcher-Djuricic V, et al. Epithelial-mesenchymal interactions in tooth germs: mechanisms of differentiation. J Biol Buccale 1983;11:173-93.

17. Zhang Y, Wang S, Song Y, et al. Timing of odontogenic neural crest cell migration and tooth forming capability in mice. Dev Dyn 2003;226:713-8.

18. Mitsiadis JA, Salmivtra M, Muramatsu T, et al. Expression of heparin-binding cytokines, midkines (MK) and HB GAM (pleiotrophin) is associated with epithelial mesenchymal interactions during fetal development and organogenesis. Development 1995;121:37-51.

19. Lehnert SA, Akhurst RJ. Embryonic expression pattern of TGF beta type-1 RNA suggests both paracrine and autocrine mechansim of action. Development 1988;104:263-73.

20. Yamashiro T, Tummers M, Thesleff I. Expression of bone morphogenetic protiens and Msx genes during root formation. J Dent Res 2003;82:172-6.

21. Osmundsen H, Landin MA, From SH, et al. Changes in gene expression during development of the molar tooth germ. Arch Oral Biol 2007;52:803-13.

22. Hall BK. Evolutionary developmental biology. 2nd ed. Berlin: Springer Publ.; 1991. pp 177-78.

23. Snead ML. Whole tooth regeneration: it takes a village of scientists, clinicians and patients. J Dent Educ 2008;72:903-911.

24. Slavkin HC, Bringas P. Epithelial-mesenchyme interactions during odontogenesis: IV. Morphological evidence for direct heterotypic cell-cell contacts. Dev Biol 1976;50:428-42.

25. Ross MH, Kaye GI, Pawlina W. Histology: a text and atlas. Philadelphia: Lippincott Williams \& Wilkins; 2003. pp 105-150.

26. Tucker AS. Salivary gland development. Semin Cell Dev Biol 2007;18:237-44.

27. Sakakura T, Nishizuka Y, Dawe CJ. Mesenchyme-dependent morphogenesis and epithelium-specific cytodifferentiation in mouse 
mammary gland. Science 1976;194:1439-41.

28. Kusakabe M, Sakakura T, Sano M, Nishizuka Y. A pituitary-salivary mixed gland induced by tissue recombination of embryonic pituitary epithelium and embryonic submandibular gland mesenchyme in mice. Dev Biol 1985;110:382-91.

29. Cutler LS. The dependent and independent relationships between cytodifferentiation and morphogenesis in developing salivary gland secretory cells. Anat Rec 1980;196:341-7.

30. Stainer P, Moore GE Genetics of cleft lip and palate: syndromic genes contribute to the incidence of non-syndromic clefts. Hum Mol Genet 2004;13:73-81.

31. Sun D, McAlmon KR, Davies JA, et al. Simultaneous loss of expression of syndecan-1 and E-cadherin in the embryonic palate during epithelial-mesenchymal transformation. Int J Dev Biol 1998;42: 733-6.

32. Grobstein C. Mechanisms of organogenetic tissue interaction. Natl Cancer Inst Monogr 1967;26:279-99.

33. Talbot LJ, Bhattacharya SD, Kuo PC. Epithelial-mesenchymal transition, the tumor microenvironment, and metastatic behavior of epithelial malignancies. Int J Biochem Mol Biol 2012;3:117-36.

34. Micalizzi DS, Farabaugh SM, Ford HL. Epithelial-mesenchymal transition in cancer: parallels between normal development and tumor progression. J Mammary Gland Biol Neoplasia 2010;15:11734.

35. Hashimoto T, Soeno Y, Maeda G, et al. Progression of oral squamous cell carcinoma accompanied with reduced E-cadherin expression but not cadherin switch. PLoS One 2012;7:e47899.

36. Dawe, CJ, Morgan, WD, Slatnick MS. Influence of epithelio-mesenchymal interactions on tumor induction by polyoma virus. Int $\mathrm{J}$ Cancer 1966;1:419-50.

37. Goldenberg DM, Pavia RA. Malignant potential of murine stroma cells after transplantation of human tumors in nude mice. Science 1981;212:65-7.

38. Schauer IG, Sood AK, Mok S, Liu J. Cancer-associated fibroblasts and their putative role in potentiating the initiation and development of epithelial ovarian cancer. Neoplasia 2011;13:393-405.

39. Kandouz M. The Eph/Ephrin family in cancer metastasis: communication at the service of invasion. Cancer Metastasis Rev 2012;31:353-73.

40. Sherbet GV. Neoplasia and cell differentiation. Embryonic and tumour cell interactions. New York: S. Karger; 1974. pp 380-396. 